\title{
Professional and Peer Social Support-Oriented mHealth App: A Platform for Adolescents with Depressive Symptomatology
}

\author{
Sayyida Masoom Gilani \\ School of Information Technology, \\ Monash University, \\ 47500 Bandar Sunway \\ Selangor, Malaysia \\ sayyida.gilani@monash.edu
}

\author{
Angeline Tanvy \\ School of Information Technology, \\ Monash University, \\ 47500 Bandar Sunway \\ Selangor, Malaysia \\ atan0064@student.monash.edu
}

\author{
Muhammad Fermi Pasha \\ School of Information Technology, \\ Monash University, \\ 47500 Bandar Sunway \\ Selangor, Malaysia \\ muhammad.fermipasha@monash.edu
}

\author{
Vanlal Thanzami \\ Department of Psychology, \\ Jeffrey Cheah School of Medicine \& Health Sciences, \\ Monash University, \\ 47500 Bandar Sunway \\ Selangor, Malaysia \\ vanlal.thanzami@monash.edu
}

\begin{abstract}
Adolescent depression has been increasing worldwide and often there are no available platforms to support them. Being one of the largest age groups of smartphone users, we investigate and implement an extension framework for mobile health (mHealth) applications to alleviate depressive symptomatology and elevate psychological well-being in adolescents. In this paper, we discuss the design and development of incorporating and integrating social support provided by professionals and peers and its effective role in a mobile context.
\end{abstract}

\section{Introduction}

Mental disorders and their negative consequences on the physical health of individuals have become a serious concern in the society [1]. Majority of the people who suffer from mental illnesses do not get any necessary treatment, especially in developing countries [2]. The onset of mental health problems is in the age of adolescence, and if left untreated, will have an impact on their adulthood and their prospective future [3]. The world health organisation (WHO) defines adolescents as individuals between the age of 10-19 [4].

Depression is a very threatening mental illness which requires constant attention and action to prevent any fatal consequences. It was reported that more than one-half of adolescent suicide victims were suffering from a depressive disorder at the time of death [5]. As the majority of adolescents own a smartphone, and they in fact, habitually spend long hours on the device [6], a mobile health (mHealth) application (app) is deemed as a suitable platform to help adolescents cope with their mental health issues, especially depression.

There are a wide range of interventions available to help adolescents cope with their depression. The most common therapeutic approach is cognitive behavioural therapy (CBT) for depressive symptomatology [7], where an individual's thoughts and behavioral patterns are improved progressively for a better quality of life. Other effective techniques include forming a self-care routine and intake of medication [8]. In this paper, we discuss incorporating professional and peer social support in the design and development of mHealth apps to alleviate depressive symptomatology and improve psychological well-being in adolescents.

The rest of the paper is organized as follows: First, we provide an overview of the mHealth apps in the mental health domain with emphasis on their strengths and limitations to set up the basis for providing an extension framework. We then present a description of the proposed framework with its features and their implementation in a mHealth app, followed by several screenshots to visually illustrate the user interface of the platform and a comprehensive guide for each page. Finally, we discuss the theoretical implications and design considerations for a sensitive age group, with conclusions and future work. 


\section{Background}

In the era of mind-body dualism, the majority of evidence-based and commercial mHealth apps are more focused on improving physical well-being whether by providing doctor consultations, management of physical fitness by means of exercise or balanced diet charts, and pharmaceutical services $[9,10,11]$. Only a few researchbased mHealth apps focus on mental health issues, in adolescents, especially depression, as indicated by comprehensive multidisciplinary systematic literature searches on several databases $[12,13,14,15]$. These reviews conclude that the vast majority of these apps promote self-help and self-management of symptoms, providing e-books, yoga and meditation coaching among some of the recommended activities. Even the top-rated apps for depression in the marketplace provide features for screening and tracking symptoms, with selfguided digitalized therapeutic interventions [16].

The common factor in these mHealth apps is the focus on self-care as they are expected to be used in adjunct to face-to-face counselling with a mental health counsellor (hereinafter, counsellor), not as an alternative $[17,18]$. However, for the geographical barriers to healthcare, which have become evident during this covid-19 pandemic [19,20], mHealth apps pose the advantage of providing improved availability, greater accessibility, increased service capacity and efficiency, minimal waiting lists, cost-effectiveness, as well as convenience. Moreover, hurdles to help-seeking such as the discomfort associated with opening up about mental health in a face-to-face environment can be reduced in a virtual setting [21,22,23,24].

To significantly improve the effectiveness of existing apps, social support by professionals and peers should be an extension. It is essential to communicate with a certified counsellor to acquire proper diagnosis and therapy. To be under care and counselling helps to address some of the issues that are felt by individuals experiencing symptoms of depression such as loneliness [25]. Additionally, peers that have been through similar experiences can provide support to each other by sharing their cognitive thoughts and behaviours. Interacting with peers can result in building a support group which may encourage expression of emotions and hasten alleviation of depressive symptoms [26].

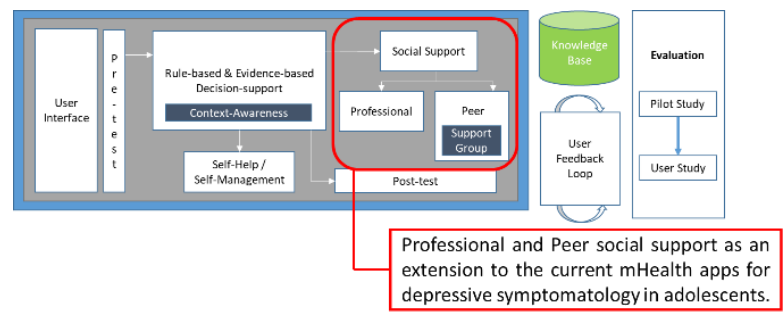

Figure 1. An extension framework for mHealth apps
Hence, professional and peer support is proposed as an extension to the existing mHealth applications for adolescents that are focused on providing low-intensity psychological interventions such as self-help and selfmanagement of depressive symptomatology. This paper describes the details of the framework and its features, along with an implementation into a distinctive mHealth app for adolescents.

\section{The proposed framework}

\subsection{Framework}

The overview of all the features proposed in the extension framework is provided in Figure 2 and Figure 3. Feature (hereinafter, F) 1 to F3 are common features for social support, while F4 to F6, in particular, are for professional support, and F7 to F9 are for peer support.

Feature 1 (F1). An emergency service that provides immediate help and safety by contacting relevant authorities when a user is severely distressed or in despair to an extent of having suicidal thoughts. With suicide being a preventable phenomenon in adolescents [27], providing an emergency service could help those in critical conditions much faster.

F1.1. The service will be initiated by clicking a button that will prompt the users to select the condition they are experiencing, after which the corresponding authorities will be contacted to the users' location. The button will be hidden on the landing page to prevent a misclick or false alarm to the authorities. As $85 \%$ of alarms are false ones, medical workers feel fatigue from it [28]; thus misclick is prevented by hiding the button. It will only be displayed if the users perform a swipe down gesture on the home page.

F1.2. Upon initiating the emergency service, the user's emergency contact will be notified with information about the critical situation for them to reach out and to their loved one's current location.

Having this emergency service feature will enable the users to get instant access to first-aid or emergency assistance, providing a straight-forward user experience whenever the need arises. In addition, users do not need to make extra effort to notify their loved ones during the crisis as the system automatically notifies the users' emergency contact when help is sought.

F2. Review the severity along with progress in depressive symptomatology each week by completing the Patient Health Questionnaire-9 (PHQ-9). Users will be able to evaluate their PHQ-9 scores at the end of each week, so they can determine the effectiveness of each action that they have performed. In addition, users can compare their scores each week to have an overall 
analysis of their improvement. Graphs and diagrams are provided to make it more comprehensible and provide an ease of understanding as self-awareness has shown to play an important role in dealing with symptoms of depression [29]. As users take the questionnaire every week, the status of their depressive symptoms will be updated and displayed, as a reminder of their recovery.

F3. Detection of specified keywords that might potentially be harmful and threatening to the user's mental well-being. This is the most important feature and a major highlight of this mHealth app. These keywords are word clusters representing depressive symptomatology and suicidal ideations [30]. The list of words detected and corresponding frequencies will be presented as a report to the counsellor. The keywords are detected and analyzed from the users' posts and chat messages. Report will be treated as confidential, which only the user and the user's counsellor will have access to.

F4. Select the right counsellor according to preference and PHQ-9 score. The mHealth app will provide numerous certified and professional counsellors with various areas of expertise, thus, the users have the option to choose accordingly. Since users' conditions are diverse based on their backgrounds and severity of their depressive symptomatology, they will have a chance to select the suitable counsellor for themselves.

F4.1. There will be a page containing a list of all the certified counsellors with their area of expertise. Every counsellor will also have a unique page containing a description of their personality, characteristics, the way they handle their sessions, and more specific details.

F4.2. Users will be able to request for a session with a counsellor that seems suitable for them. Upon choosing their counsellor, users will be 'assigned' to the counsellor and they can conduct their one-to-one sessions personally. The users can view a detailed list of all previous and current sessions on their profile.

This feature is introduced as a therapeutic relationship is crucial when it comes to counselling despite the quality of the session itself [31]. Having an open conversation, and trusting each other affects the result of the session by a large margin. The connection that is formed between a counsellor and user is what matters in the philosophy of therapy [32], therefore, this feature will enhance the ability of users to choose the right therapist for them.

F5. A communication platform for user-counsellor alliance. In order to converse with counsellors, there is a private chat feature which allows users and counsellors to send messages back and forth. In the chat room, users can send files in any form: image, video, audio, pdf, and others formats. With implementation of voice call and video call features, users can have a virtual face-to-face communication with counsellors to form a good-quality therapeutic relationship. By using voice call and video call, users can get a deeper understanding of their situations and symptoms of ailing mental health [33]. Another purpose is to overcome the geographical barriers that exist between counsellors and users [34]. Besides, interacting with professionals reduces the feeling of isolation, and positively impacts one's lifestyle [35].

F6. Leave reviews, comments and feedback for the counsellor at the end of the session. Users can evaluate a counselling session either negatively or positively upon completion. This will help other users to have a glimpse into the counsellor's approach and also be beneficial for counsellors to reflect and improve their subsequent sessions.

F6.1. Once the user-counsellor communication has ended, users will be asked to review the counsellor's session in terms of the session-flow, helpfulness, suggestions for future improvements and other relevant details. Upon submission, however, before posting, the comments will be sent to a moderator for screening and accordingly removing feedback with inappropriate language use (e.g., abusive words).

F6.2. All the reviews, comments and feedback will be posted on the corresponding counsellor's page on F4.1 after moderation.

In addition to the details of the counsellors from their respective page on F4.1., the users can also view the description of a counsellor's session from the perspective of other users. This way, users can select a session that is appropriate and relevant for them. Counsellors could also receive feedback and self-reflect to better conduct the future sessions, as counsellors like to know their performance results of the sessions they have conducted so far. They take it as an educational opportunity and manifest their learning in various ways for the following sessions [36].

F7. Creation of profile and managing friends for each user. Each user will have a profile for the online social community to interact with other users. Users will be asked to fill in the basic information to build up a profile which can be updated at any time. This will help counsellors to get a brief summary of the users before the session and also for users to recognize their peers that are using the mHealth app. Users are also able to send friend requests to their peers, so that they can see each other's posts and also chat privately as described in F9.

F8. Interactive social media feed (hereinafter, feed) that will allow users to view the posts of their friends in real-time. In UK 2020, it was reported that $90 \%$ of adolescents aged 11 and above own mobile phones [37] 
and $72 \%$ of them use Instagram [38]. Therefore, having a social-media-like platform to let users share their ideas and thoughts would better help them cope with their depressive symptoms. Moreover, by having a feed to share their experiences, users will feel connected and supported which is expected to result in alleviation of their depressive symptomatology [39].

F8.1. There will be a feed containing the posts of the user's friends expressing their emotions. Users will be able to see the author of the post, the content, and the time stamp. Moreover, users will be able to interact with the posts to share their opinions.

F8.2. The contents of the posts could be in textual, visual (image and video) and audio file format. Users may post content with either or any of these combinations.

F8.3. Users will be able to like or dislike a post.

F8.4. Any negative, threatening, and harmful posts will be detected using an intelligent engine and reported to the current user's counsellor, as it is important to prevent any negativity in an online community [40].

This platform will increase the accessibility to receiving timely peer support, and for users to share their emotions and feelings multiple times in a day. This is in turn believed to bring positive effects towards reducing symptoms of depression [41].

F9. Private messaging among users in the mHealth app. Users can have a private conversation with others with an end-to-end encryption. By doing so, users are able to have a one-on-one chat with more freedom with specific individuals. This platform has the same structure as F4, enabling users to have private conversations via texting or direct-communication (voice calls and video calls) and sharing files. The purpose of this feature is to bring peers together, to perceive empathy, respect and support among users [42]. Hence, it is expected that this feature would have a positive influence on the depressive symptomatology.

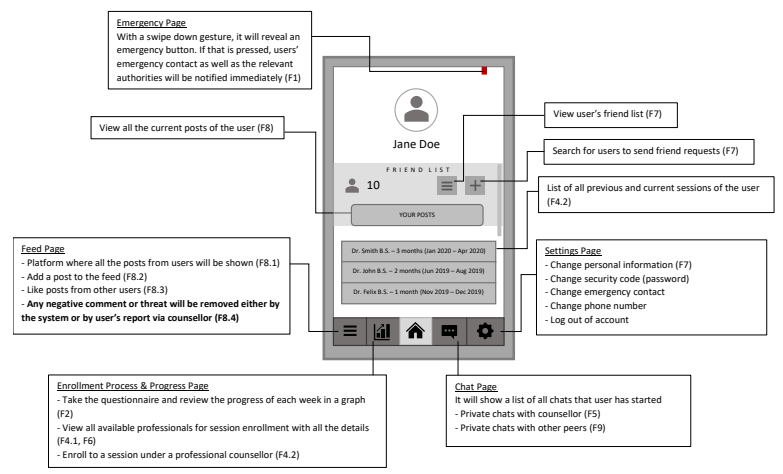

Figure 2. User's landing page

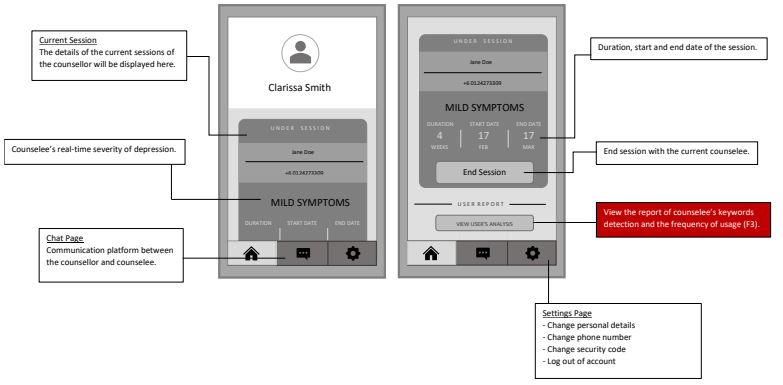

Figure 3. Counsellor's landing page

\subsection{Assessment of depressive symptomatology}

PHQ-9 detects the presence of depression and grades the severity of symptoms in medical and primarily mental health settings. It is an easy-to-use selfadministered questionnaire with nine questions and requires about three minutes to complete. PHQ-9 also assesses suicidal risks and self-harm, which are important indicators for the targeted mHealth app. Moreover, PHQ-9 has often been used in depression assessment and management apps and other programs related to this mental health problem [43,44,45]. Therefore, with evidence of its stability and reliability, it is the most suitable tool for measuring symptoms of depression in this mHealth app targeted for adolescents.

\subsection{Programming environment}

For the mHealth app, the software used for frontend and back-end development is Android Studio. It is an integrated development environment (IDE) for Google's Android operating system, which uses Kotlin, Java and $\mathrm{C}++$ as its programming languages. During the development of this app, the back-end was fully coded in Java, as it is one of the most relevant to the objectoriented programming environment. For the front-end, $\mathrm{XML}$ is the only method provided by Android Studio.

For the database, Firebase is used mainly because it is a real-time database which allows all the data to synchronize instantaneously (storing and retrieving), when creating a profile, posting content on the feed, or changing account settings. It serves as a great advantage for this development, specifically for F5 and F9, which allows users to communicate in real time. Firebase also has several built-in functions like authentication and verification for registration and login. Moreover, setting up a connection between Android Studio and Firebase is straightforward. It is also relatively effortless to access, modify, add, and delete data from Firebase in Android Studio, thus, making the coherence between the two quintessential for this mHealth app. 


\section{User interface}

The landing page for both users and counsellors will be a welcome page with the same interface. Two options will be provided; login for a registered user, or, signup if the user does not already have an account. Both the buttons lead to their respective pages; the login page requires the same details from the users and counsellors (email and password). For counsellors, they need to check the "I am a counsellor" box, only after which they will be able to log in or register in the mHealth app.
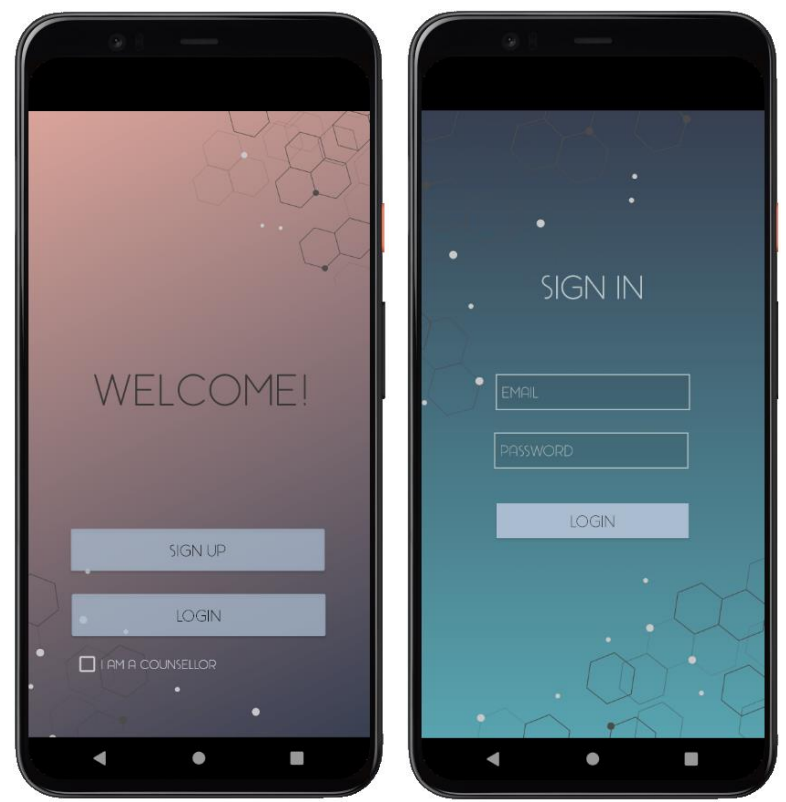

Figure 4. Welcome page and login page

To register, both users and counsellors will be asked to fill up several details and take questionnaires, which will also be used for the vetting process. Unlike the login page, the information procured will be different for signing up; for the users, it will be focused on their contact details, and severity of depressive symptoms, which will be measured by the PHQ-9 questionnaire. A user will be allowed to sign up only if the date of birth (DOB) keyed in matches the predefined age group of adolescence. Additionally, users' emergency contact details will be obtained during registration for use in F1. As for the counsellors, information will be gathered on their area of expertise and details of their sessions. For added security, at the end of registration, after setting up a desired password for the account, every user will need to verify their phone number by keying in a one-time password (OTP) sent to their personal device. Moreover, for counsellors, there is an additional step of email verification in place for a moderator to ensure the registration of licensed mental health counsellors.

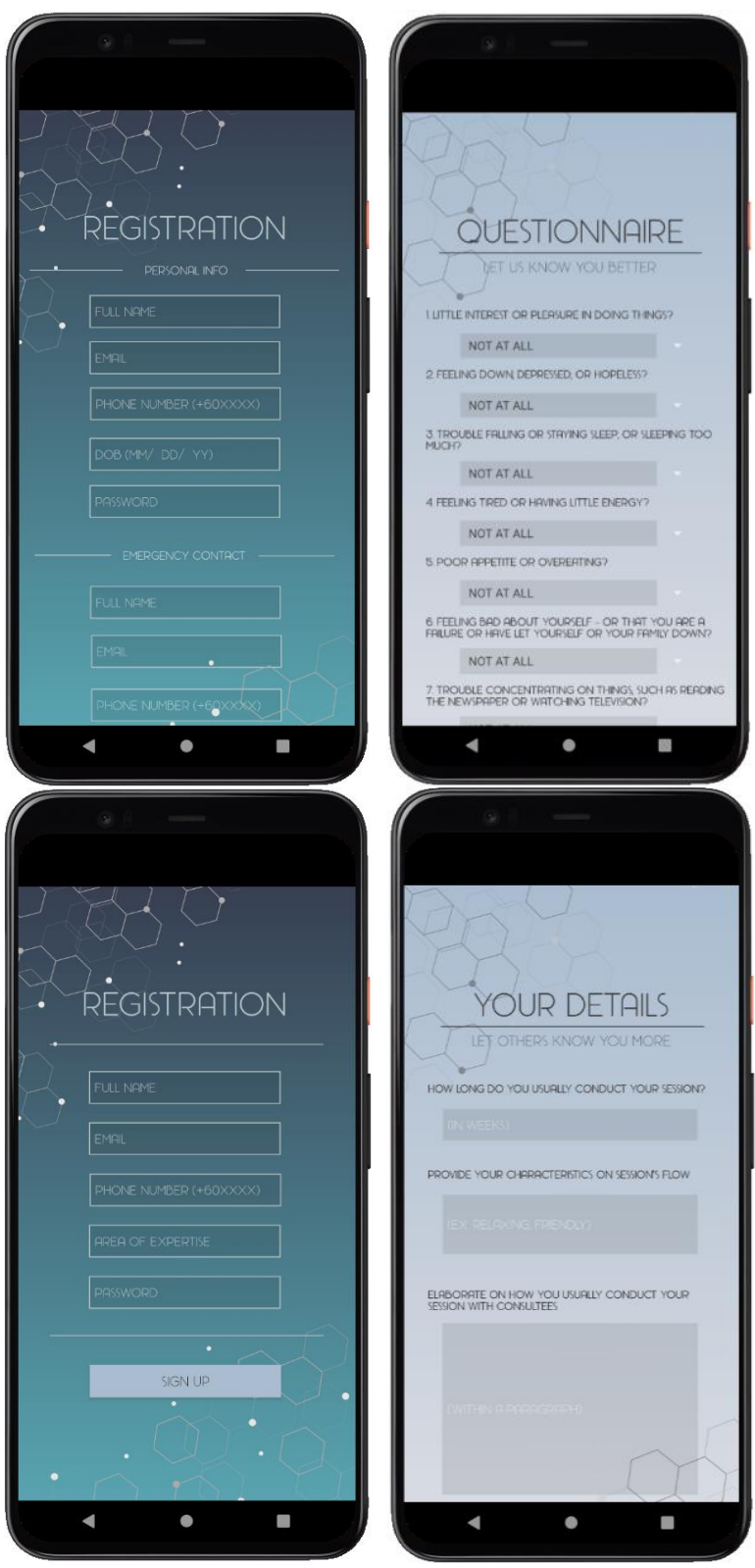

Figure 5. Registration pages

\subsection{Users' account}

Upon logging in, the navigation bar provided at the bottom of the screen can be used as a guide to move between the app pages. As shown in Figure 6, users can view their friends' posts on the feed which is located on the leftmost side of the navbar. The posts will be sorted by descending date, with the latest post displayed first. Users can like a post by using the corresponding button on bottom left corner.

The add button on the bottom right of the screen can be used to add posts, which will initiate a popup querying the user for the post content. 

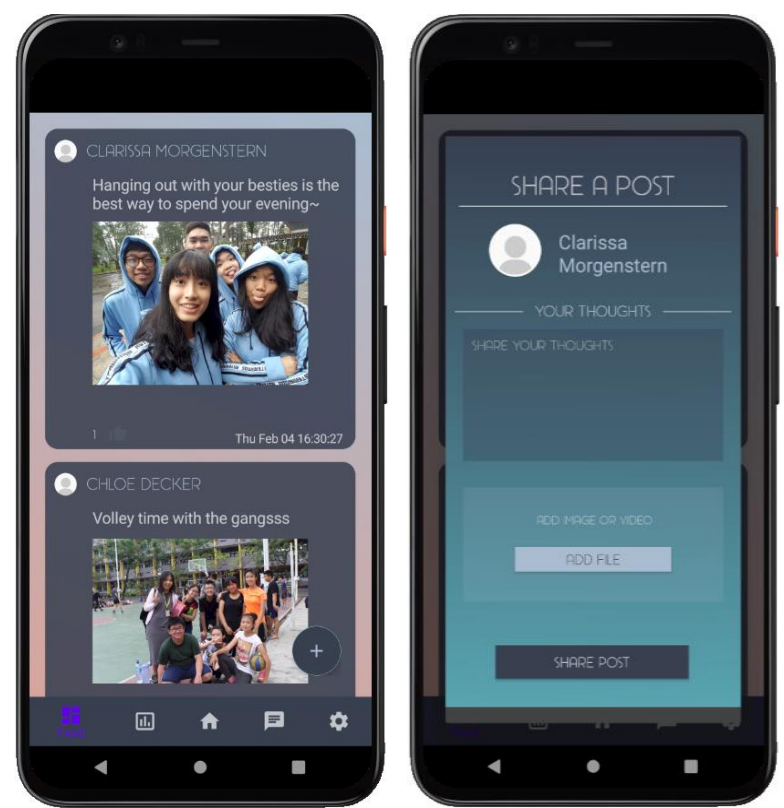

Figure 6. Feed page and add post dialog

The progress page displays a graph through which the users can track their improvement in the PHQ-9 score and severity of depressive symptoms, with an appropriate color such as green for indicating no symptoms, orange for 'moderate symptoms', and so on. The questionnaire can also be taken again and details of ongoing sessions will be displayed on this page.

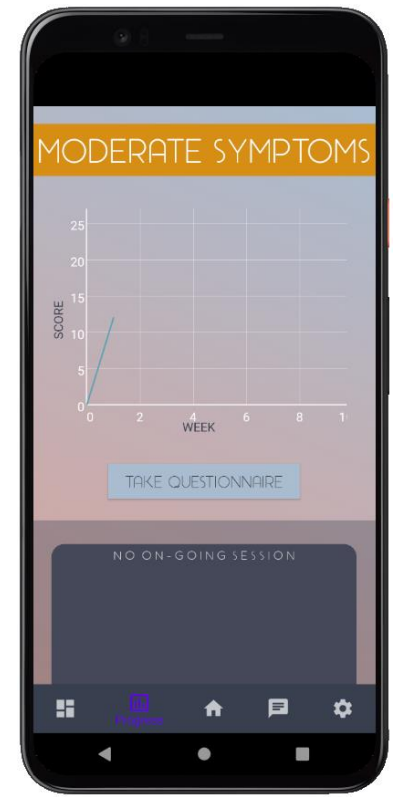

Figure 7. Progress page

From the progress page, the user can enroll in a counsellor's session by clicking the 'enroll to a session' button, which shows a list of counsellors with their expertise. The user can click on the counsellor to see more details in a dialog. If a counselling session is required, the "request session" button can be used to send a request to the counsellor, and the user will need to wait for confirmation.

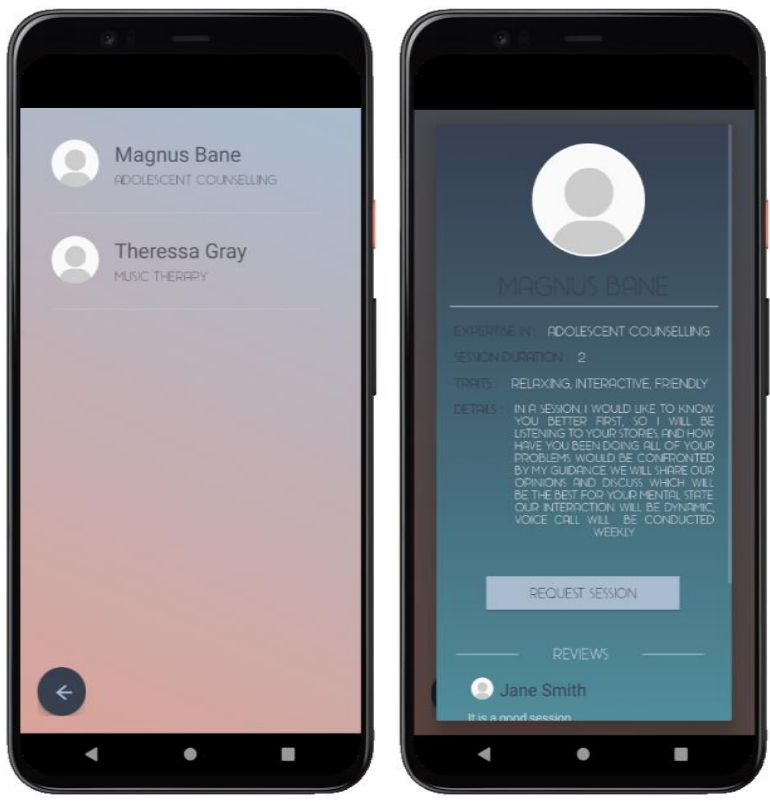

Figure 8. Enrollment process

Figure 9 will be the user's landing page upon logging in. The emergency button is located at the top of the screen. As mentioned in F1, it will be hidden, and only displayed if the screen is pulled down by a swipe gesture. Users can see their previous posts by clicking on the 'your posts' button. The corresponding buttons for add a friend and list of friends are also available on this page. At the bottom of the page, the history of all the counselling sessions of the user is displayed.
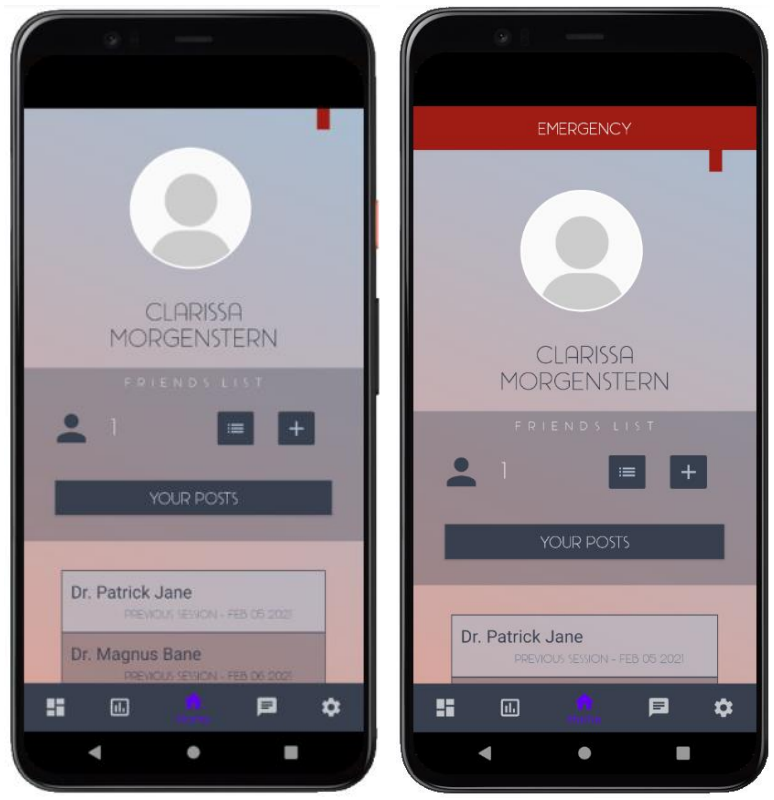

Figure 9. Home page 
The interface of the chat page is the same for peerto-peer private messaging and for communication with counsellors. Clicking on the peer's or counsellor's name will bring the user to a private chat room. Inside, the user can send messages and files. Voice and video call can also be initiated by clicking the corresponding button on the top right of the screen. The verbal and visual communication window is given a unique ID. The counsellor and user can choose from multiple options to turn the video on or off, mute the call, and end it.
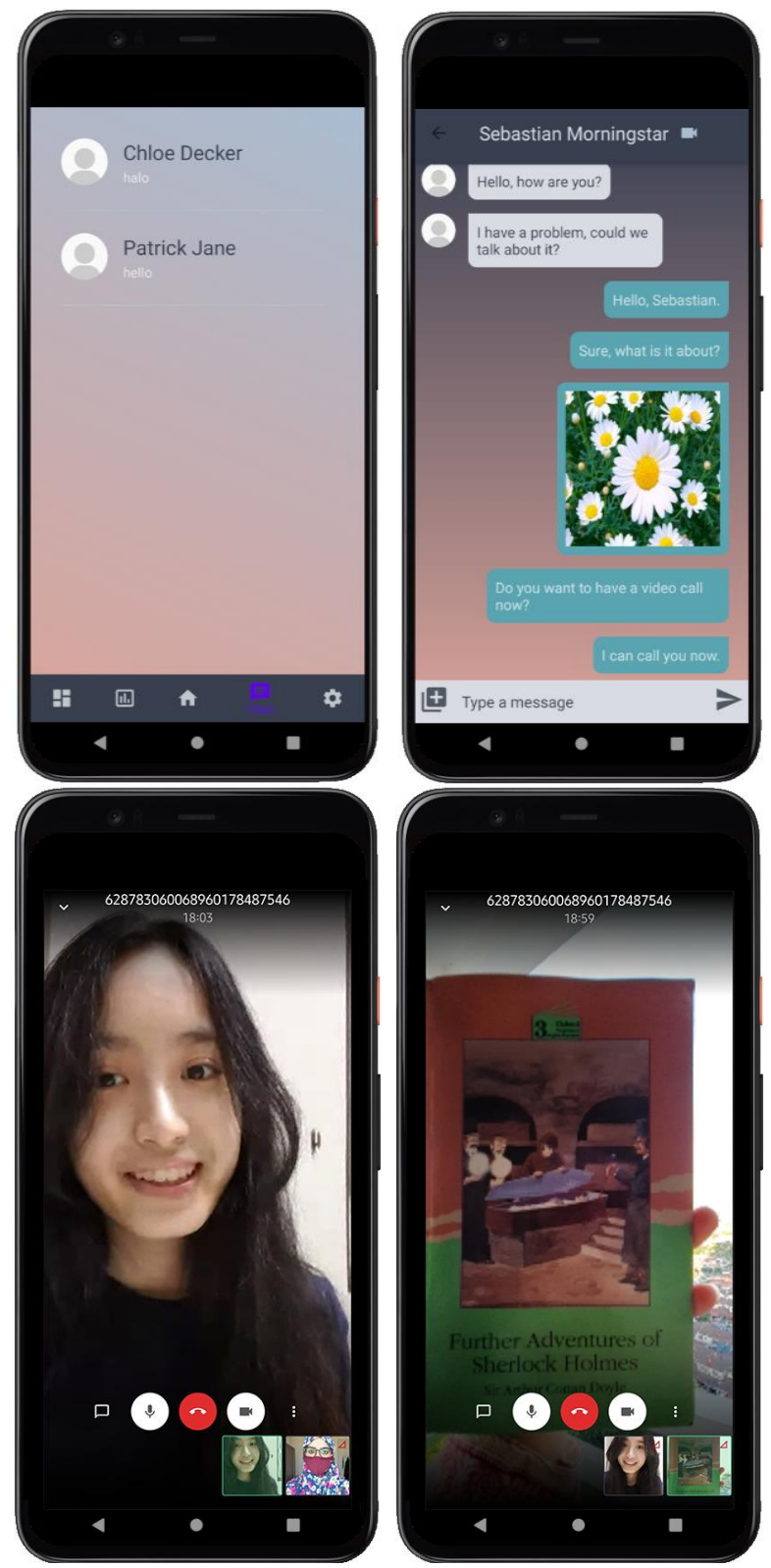

Figure 10. Communication pages

The settings page is for users to change their details. A dialog will pop up after the user clicks on any of the categories that they want to change, then the desired information can be updated accordingly. To prevent any unintentional or unauthorized changes, users are asked to key in their password for confirmation.
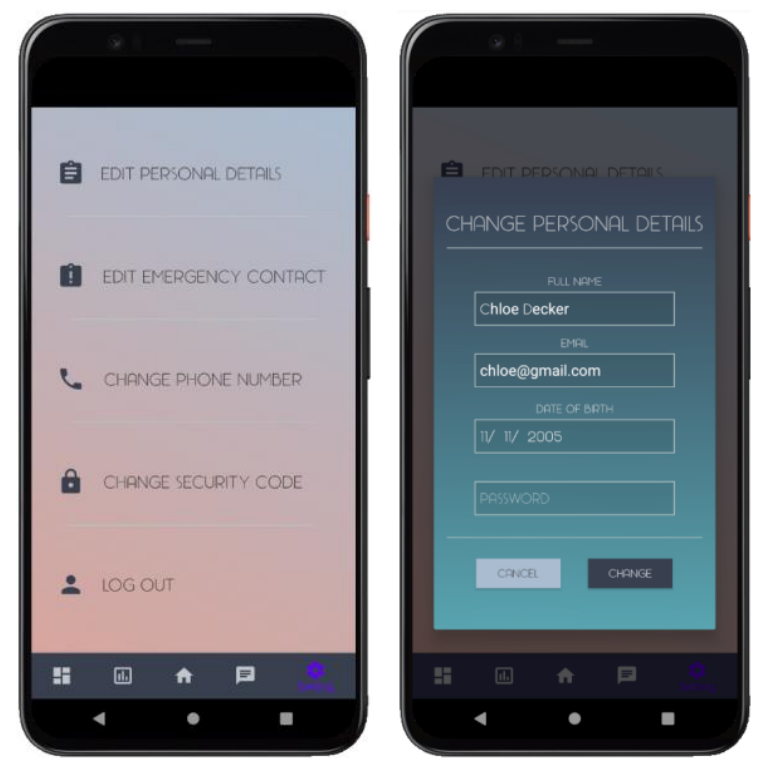

Figure 11. Settings page and edit details dialog

\subsection{Counsellors' account}

The counsellor' accounts will have 3 pages. The landing page is for viewing, accepting and managing session requests. The chat page and settings page will have the same interface and functionalities as the users' accounts. Additionally, counsellors can view keyword analytics of the ones under their care. It will be presented in the form of a report displaying the alarming words used and their number of occurrences.
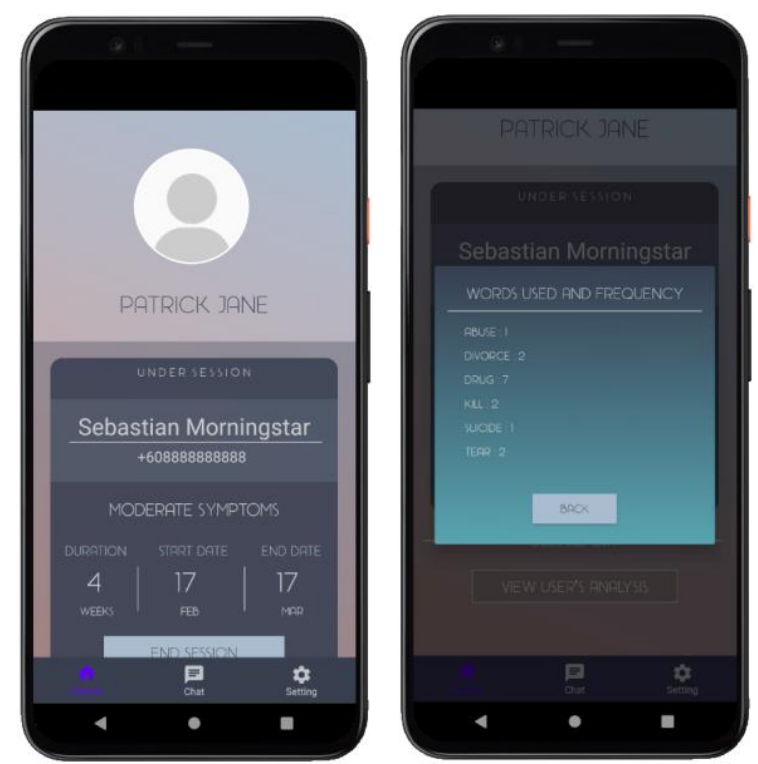

Figure 12. Counsellors' pages 


\section{Discussion}

This paper describes incorporation and integration of social support provided by professionals and peers in the design and development of mHealth apps for depressive symptomatology in adolescents. With a wide gap between the supply and demand of treatment for adolescents, this platform could circumvent the barriers and offer timely mental healthcare [46]. However, the treatment gap can also be potentially addressed by other digital health interventions (DHIs), whether delivered online (web-based) or offline (SMS, CD-ROM). Webbased programs are deemed effective for a wide range of mental illnesses in adolescents, including depression $[24,47]$.

With the constant evolution of technology, over the last decade, adoption of mobile phones in particular has increased at a fast pace [14]. Smartphone penetration in adolescents has increased rapidly with over $90 \%$ of the population owning a device, regardless of their sociodemographic domain and these upward trends are continuing [48]. Therefore, taking a mHealth approach to adolescent depression is ideal in real-world settings. Besides, aligning the tasks (features of the framework) and technology (mHealth app) characteristics result in better performance at the tasks and overall utilization of the technology, as per the theory of task-technology fit [49]. With features that require characteristics present in modern-day smartphones such as enabling location for emergency services, conveniently capturing images or videos, and recording audios, a mHealth app can ease and expand the access to mental health treatments.

This is supported by the perceptions of adolescents and counsellors on mHealth apps for mental health. With the strong uptake of smartphones, adolescents are receptive to using apps for their mental well-being and counsellors are show a positive response on providing professional support via online platforms. Adolescents perceive social interactions (peer social support) as a key factor that influences use of apps, in addition to accessibility, functionality, realism and engagement [50]. This finding suggests that when social support is offered in a mHealth platform, it is likely to be utilized. However, in the app description, users will be notified of the expectations to use the app frequently to both provide and receive social support.

For both stakeholders, ethical and legal regulations affect uptake of any e-Health platforms, including mHealth apps especially for a sensitive subject like depression. The most common theme that has emerges as a downside to using mHealth apps is 'safety'. However, experts and end-users have responded to possible approaches to addressing these concerns, including confidentiality in communication, selective interaction, and moderation for cyber-bullying [50].
Based on these recommendations, the critical design considerations in the extension framework include offering end-to-end encryption for the all the features related to communication and giving users a control over the audience of their social media posts (by sending friend requests). Besides, social media is moderated by professionals with the feature for keyword detection and extraction within all textual communication of users. However, there is a limitation as only words that are deemed threatening, and harmful are considered, as opposed to analyzing the post contents holistically. If a user posts 'I am feeling suicidal' and uses the same trigger word in a non-first-person context such as 'My friend, Liza, is suicidal as her dentist appointment was cancelled', the counsellor will see two occurrences of the same keyword in the report. Besides, non-textual data such as a post with a triggering image could go unnoticed if not captioned (using text).

\section{Conclusion and future work}

In this paper, we have presented an extension framework and its implementation as a mHealth app for providing professional and peer social support to adolescents with depressive symptomatology, which contributes to the knowledge of ICT-enabled (mHealth) self-management of chronic diseases and conditions. As shown in Figure 1, this is part of a large-scale project aiming to explore the role of mHealth apps for mental health in this vulnerable age. A pilot test and user study of the platform is planned for the next phase of the project. This research will undergo ethical scrutiny via the human research ethics committee, which takes into account factors such as consent (self and/or parental), possible benefits and risks, data collection and retention, and confidentiality to ensure protection of welfare and rights of participants.

The pilot test will examine the mHealth app in terms of the interface design with HCI experts, counsellors and an adult population. These different categories of participants will be invited for focus groups to interact with the app and provide constructive and critical feedback on its design. Based on the identified limitations, and the feedback from focus groups, the design framework will be refined and its implementation will then be validated in a real-world setting with adolescents and counsellors, in addition to moderators.

\section{References}

[1] K. Naicker, N. L. Galambos, Y. Zeng, A. Senthilselvan, and I. Colman, "Social, demographic, and health outcomes in the 10 years following adolescent depression," J Adolesc Health, vol. 52, no. 5, pp. 533538, 2013. 
[2] A. F. Jorm, S. B. Patten, T. S. Brugha, and R. Mojtabai, "Has increased provision of treatment reduced the prevalence of common mental disorders? Review of the evidence from four countries," World Psychiatry, vol. 16, no. 1, pp. 90-99, 2017.

[3] A. Petito et al., "The Burden of Depression in Adolescents and the Importance of Early Recognition", The Journal of Pediatrics, vol. 218, pp. 265-267.e1, 2020.

[4] World Health Organization, "Adolescent data.", who.in, 2021 [online].

[5] K. Hawton and K. van Heeringen, "Suicide", The Lancet, vol. 373, no. 9672, pp. 1372-1381, 2009.

[6] J. Jacobo, "Teens spend more than 7 hours on screens for entertainment a day: Report", abcNEWS, 2019.

[7] K. Kamenov, M. Cabello, M. Nieto, R. Bernard, E. Kohls, C. Rummel-Kluge, and J. L. Ayuso-Mateos, "Research Recommendations for Improving Measurement of Treatment Effectiveness in Depression," Frontiers in Psychology, vol. 8, p. 356, 2017.

[8] A. Qahtani A and A. Qahtani N, "Prevention of Depression: A Review of Literature," Journal of Depression and Anxiety, vol. 6, no. 4, 2017.

[9] A. Chebanova, "Top Mobile Healthcare Applications on the Market Today", Steelkiwi.com, 2021. [Online].

[10] S. Morse, M. Murugiah, Y. Soh, T. Wong and L. Ming, "Mobile Health Applications for Pediatric Care: Review and Comparison", Therapeutic Innovation \& Regulatory Science, vol. 52, no. 3, pp. 383-391, 2018.

[11] A. Lee et al., "Efficacy and Effectiveness of Mobile Health Technologies for Facilitating Physical Activity in Adolescents: Scoping Review", JMIR mHealth and uHealth, vol. 7, no. 2, p. e11847, 2019.

[12] R. Grist, J. Porter, and P. Stallard, "Mental Health Mobile Apps for Preadolescents and Adolescents: A Systematic Review," Journal of Medical Internet Research, vol 19, no. 5, p. e176, May 2017.

[13] J. K. Das, R. A. Salam, Z. S. Lassi, M. N. Khan, W. Mahmood, V. Patel, and Z. A. Bhutta, "Interventions for Adolescent Mental Health: An Overview of Systematic Reviews," J Adolesc Health, vol. 59, no. 4, pp. S49-S60, 2016.

[14] R. Grist, A. Croker, M. Denne, and P. Stallard, "Technology Delivered Interventions for Depression and Anxiety in Children and Adolescents: A Systematic Review and Meta-analysis," PubMed.gov, vol. 22, no. 2, pp. 147-171, Jun. 2019.

[15] M. Punukollu and M. Marques, "Use of mobile apps and technologies in child and adolescent mental health: a systematic review", Evidence Based Mental Health, vol. 22, no. 4, pp. 161-166, 2019.

[16] C. Qu, C. Sas, C. Daudén Roquet and G. Doherty, "Functionality of Top-Rated Mobile Apps for Depression: Systematic Search and Evaluation", JMIR Mental Health, vol. 7, no. 1, p. e15321, 2020.

[17] M. Price et al., "mHealth: A Mechanism to Deliver More Accessible, More Effective Mental Health Care", Clinical Psychology \& Psychotherapy, vol. 21, no. 5, pp. 427-436, 2014.

[18] K. Weisel, L. Fuhrmann, M. Berking, H. Baumeister, P. Cuijpers and D. Ebert, "Standalone smartphone apps for mental health - a systematic review and meta-analysis", npj Digital Medicine, vol. 2, no. 118, 2019.

[19] R. Bashshur, C. Doarn, J. Frenk, J. Kvedar and J. Woolliscroft, "Telemedicine and the COVID-19
Pandemic, Lessons for the Future", Telemedicine and $e$ Health, vol. 26, no. 5, pp. 571-573, 2020.

[20] D. Giansanti and I. Aprile, "Letter to the Editor: Is the COVID-19 Pandemic an Opportunity to Enlarge the Telemedicine Boundaries?", Telemedicine and e-Health, vol. 26, no. 9, pp. 1123-1125, 2020.

[21] A. Barak and J. Grohol, "Current and Future Trends in Internet-Supported Mental Health Interventions", Journal of Technology in Human Services, vol. 29, no. 3, pp. 155-196, 2011.

[22] G. Clarke and B. Yarborough, "Evaluating the promise of health IT to enhance/expand the reach of mental health services", General Hospital Psychiatry, vol. 35, no. 4, pp. 339-344, 2013.

[23] D. Erbe, H. Eichert, H. Riper and D. Ebert, "Blending Face-to-Face and Internet-Based Interventions for the Treatment of Mental Disorders in Adults: Systematic Review", Journal of Medical Internet Research, vol. 19, no. 9 , p. e306, 2017.

[24] C. Hollis et al., "Technological innovations in mental healthcare: harnessing the digital revolution", British Journal of Psychiatry, vol. 206, no. 4, pp. 263-265, 2015.

[25] [10]G. Arslan, "School belongingness, well-being, and mental health among adolescents: exploring the role of loneliness", Australian Journal of Psychology, vol. 73, no. 1, pp. 70-80, 2021.

[26] P. Pfeiffer, M. Heisler, J. Piette, M. Rogers and M. Valenstein, "Efficacy of peer support interventions for depression: a meta-analysis", General Hospital Psychiatry, vol. 33, no. 1, pp. 29-36, 2011.

[27] M. de Agrela Gonçalves Jardim, R. Silva, G. da Silva Junior, J. Caldas, Z. Santos and I. Silva, "Suicide: A Preventable Phenomenon in Adolescents", Psychology, vol. 08, no. 07, pp. 1008-1018, 2017.

[28] "K. Gaines, "Alarm Fatigue is Way Too Real (and Scary) For Nurses," nurse.org, 2019.

[29] A. Kranzler, J. Young, B. Hankin, J. Abela, M. Elias and E. Selby, "Emotional Awareness: A Transdiagnostic Predictor of Depression and Anxiety for Children and Adolescents", Journal of Clinical Child \& Adolescent Psychology, vol. 45, no. 3, pp. 262-269, 2015.

[30] R. N. Grant, D. Kucher, A. M. Leon, J. F. Gemmel, D. S. Raicu, and S. J. Fodeh, "Automatic extraction of informal topics from online suicidal ideation", $B M C$ Bioinformatics, vol. 19, p. S8, 2018.

[31] J. Fraga, The Importance of Choosing the Right Therapist, psyched.

[32] M. Nor, "Counselling: What and How", Counseling and Therapy, IntechOpen, 2020

[33] H. Hewitt, J. Gafaranga, and B. H. Mckinstry, "Comparison of face-to-face and telephone consultations in primary care: Qualitative analysis," British Journal of General Practice, vol. 60, no. 574, pp. e201-e212, 2010.

[34] E. De Weger, D. Macinnes, J. Enser, S. Francis and F. Jones, "Implementing video conferencing in mental health practice", Journal of Psychiatric and Mental Health Nursing, vol. 20, no. 5, pp. 448-454, 2012.

[35] K. Krause, N. Midgley, J. Edbrooke-Childs and M. Wolpert, "A comprehensive mapping of outcomes following psychotherapy for adolescent depression: The perspectives of young people, their parents and therapists", European Child \& Adolescent Psychiatry, 2020.

[36] H. Brattland, J. Høiseth, O. Burkeland, T. Inderhaug, P. Binder and V. Iversen, "Learning from clients: A qualitative investigation of psychotherapists' reactions to 
negative verbal feedback", Psychotherapy Research, vol. 28, no. 4, pp. 545-559, 2016.

[37] "Most children own mobile phone by age of seven, study finds," The Guardian, 2020.

[38] J. Chen, "Social media demographics to inform your brand's strategy in 2020," sproutsocial, 2020.

[39] S. Rice et al., "Online and Social Networking Interventions for the Treatment of Depression in Young People: A Systematic Review", Journal of Medical Internet Research, vol. 16, no. 9, p. e206, 2014.

[40] K. Griffiths, J. Reynolds and S. Vassallo, "An Online, Moderated Peer-to-Peer Support Bulletin Board for Depression: User-Perceived Advantages and Disadvantages", JMIR Mental Health, vol. 2, no. 2, p. e14, 2015.

[41] L. D. McColl, P. Rideout, T. N. Parmar, and A. A. Aji, "Peer Support Intervention Through Mobile Application: An Integrative Literature Review and Future Directions," American Psychology Association, vol. 55, no. 4, pp. 250-257, Nov. 2014

[42] N. Stapper, G. Benavides Jimenez, Y. Barenco Abbas and M. Homsy King, "Mind Support: A Systematic Review of Online Peer Support Platforms", Applied Neuroscience and Mental Health, vol. 1, no. 1, pp. 2-20, 2021.

[43] K. L. Smarr, and A. L. Keefer, "Measures of depression and depressive symptoms: Beck Depression Inventory-II (BDI-II), Center for Epidemiologic Studies Depression Scale (CES-D), Geriatric Depression Scale (GDS), Hospital Anxiety and Depression Scale (HADS), and Patient Health Questionnaire-9 (PHQ-9)," Wiley Online Library, vol. 63, no. 11, pp. S454-456, 2011.
[44] P. A. Arean, K. A. Hallgren, J. T. Jordan, A. Gazzaley, D. C. Atkins, P. J. Heagerty, et al. "The Use and Effectiveness of Mobile Apps for Depression: Results From a Fully Remote Clinical Trial," Journal of Medical Internet Research, vol. 18, no. 12, p. e330, 2016.

[45] N. Shen, M. J. Levitan, A. Johnson, J. L. Bender, M. H. Page, A. R. Jadad, et. al. "Finding a Depression App: A Review and Content Analysis of the Depression App Marketplace," JMIR mHealth and uHealth, vol. 3, no. 1, p. e16, 2015.

[46] S. Neufeld, V. Dunn, P. Jones, T. Croudace and I. Goodyer, "Reduction in adolescent depression after contact with mental health services: a longitudinal cohort study in the UK", The Lancet Psychiatry, vol. 4, no. 2, pp. 120-127, 2017.

[47] C. Hollis et al., "Annual Research Review: Digital health interventions for children and young people with mental health problems - a systematic and meta-review", Journal of Child Psychology and Psychiatry, vol. 58, no. 4, pp. 474-503, 2017.

[48] M. Dubad, C. Winsper, C. Meyer, M. Livanou and S. Marwaha, "A systematic review of the psychometric properties, usability and clinical impacts of mobile moodmonitoring applications in young people", Psychological Medicine, vol. 48, no. 2, pp. 208-228, 2018.

[49] R. Spies, S. Grobbelaar and A. Botha, "A Scoping Review of the Application of the Task-Technology Fit Theory", Lecture Notes in Computer Science, pp. 397408, 2020.

[50] R. Kenny, B. Dooley and A. Fitzgerald, "Developing mental health mobile apps: Exploring adolescents' perspectives", Health Informatics Journal, vol. 22, no. 2, pp. 265-275, 201 